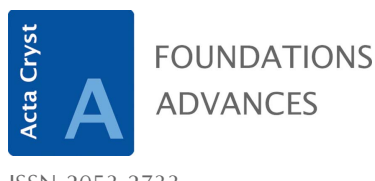

ISSN 2053-2733

\section{Prime Numbers and the Riemann Hypothesis. By Barry Mazur and William Stein. Cambridge University Press, 2016. Pp. xi + 142. Price GBP 17.99 (paperback). ISBN 9781107499430.}

\author{
Massimo Nespolo*
}

Université de Lorraine, CRNS, CRM2, Nancy, France. *Correspondence e-mail: massimo.nespolo@univ-lorraine.fr

Prime Numbers and the Riemann Hypothesis is an agile, unusual book written over a decade, one week per year; it can be considered a sort of collaborative work, in that each version was put online with the purpose of getting feedback. As explicitly stated in the preface, the final version owes a lot to the readers. In a 'fast-food' world where publishing has become a race but where first place doesn't always correspond to the best in terms of content, this 'slow-food' approach is highly commendable.

As the title clearly indicates, the book is an introduction to the Riemann hypothesis, a conjecture about the number of primes less than a given number, sometimes considered the most important unresolved problem in pure mathematics. Throughout the book, we get three different formulations of the Riemann hypothesis (called 'first', 'second' and 'fourth': I wonder if I have missed the 'third', despite repeated browsing back and forth through the book), which range from a rather intuitive approach to the most modern and rigorous statement. Despite its small size, the book is divided into 38 chapters which form four parts. Each chapter is extremely short and succinct (one to six pages), and includes a series of endnotes to where the few derivations are relegated. The purpose is clearly pedagogical and the book is suitable for readers with minimal mathematical background, although the mathematical content increases from the first to the fourth part.

Part I (24 chapters out of 38) gives a historical introduction to the problem of counting prime numbers and the functions that approximate the staircase of primes. In Chapter 13 we get the first formulation of the Riemann hypothesis: the number of primes less than $X$ is approximately $\operatorname{Li}(X)$, the area of the region from 2 to $X$ under the graph of $1 / \log (X)$. And in Chapter 19 we get the second formulation, according to which the staircase of primes can be approximated by a $45^{\circ}$ straight line. Before the end of this part, we get an introduction to Fourier analysis which is seen as a bridge relating music files, data compression and prime numbers (a nice pedagogical approach which a crystallographer would certainly complete with the scattering of radiation by a periodic grid). I noted a curious mistake which escaped the long gestation of this book. In Chapter 10, Carl Friedrich Gauss is made half a century younger: he is said to have lived from 1824 to 1908 instead of 1777-1855. Out of curiosity, I tried to find out who these birth and death dates might belong to, but none of the hits was relevant to this topic.

Part II (six chapters) is devoted to distributions (generalized functions), with special attention to the Dirac $\delta$ function and its Fourier transform. It is meant as a hors d'oeuvre for the plat de résistance, coming in the next two parts, devoted to the analysis of the Riemann hypothesis.

Part III (five chapters) introduces the Riemann spectrum, a discrete sequence of positive real numbers $\theta_{i}$ marking the peaks of trigonometric series, which turn out to be the key to the placement of prime numbers on the line of integer numbers.

Part IV (three chapters) completes the journey by introducing the zeta function whose zeros are related to the Riemann spectrum: the last formulation of the Riemann hypothesis is here in terms of the nontrivial zeros of the zeta function as $\frac{1}{2} \pm \eta \theta_{i}$, where $\theta_{i}$ precisely comprises the spectrum of primes.

Clearly, this small book is not sufficient for an in-depth analysis of the Riemann hypothesis, and never pretended to be. It is a nice introduction to the topic which serves 


\section{book reviews}

as an invitation for a more detailed analysis. Nevertheless, some more effort could have been made; for example, a couple of examples following definition 29.1 of spike would certainly have been welcome.
The index spans only two pages and is of limited help. While the attentive reader would benefit from a more detailed index, the small size of the book means that searching through it is not an impossible task. 